\title{
Pluralities, Collectives, and Composites
}

\author{
Claudio MASOLO ${ }^{\text {a }}$, Laure VIEU ${ }^{\text {b,a }}$, Roberta FERRARIO ${ }^{\text {a }}$, Stefano BORGO ${ }^{\text {a }}$, \\ Daniele PORELLO ${ }^{\text {a }}$

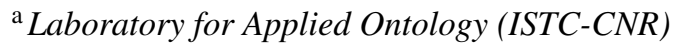 \\ b IRIT, CNRS, Toulouse University
}

\begin{abstract}
Forests, cars and orchestras are very different ontological entities, and yet very similar in some aspects. The relationships they have with the elements they are composed of is often assumed to be reducible to standard ontological relations, like parthood and constitution, but how this could be done is still debated. This paper sheds light on the issue starting from a linguistic and philosophical analysis aimed at understanding notions like plurality, collective and composite, and proposing a formal approach to characterise them. We conclude the presentation with a discussion and analysis of social groups within this framework.
\end{abstract}

Keywords. mereology, parthood, constitution, membership, plural, plurality, collective, composition, composite, social group, agency, functionality.

\section{Introduction}

In most domains, applied ontology is bound to deal with groups or collections-roughly, entities such as orchestras, herds, forests or decks of cards that can be said to have members and that we here call collectives. However, only few formal ontology theories attempt to deeply characterise collectives $[1,2,3]$. Focusing on objects (aka endurants or continuants), we propose a new account of collectives contrasting these objects with those that we call composites (aka assemblies [4] or complexes [5]), i.e., objects such as animals, trees, mountains or cars that have another sort of internal structure.

The linguistic literature on plurals and group (or collection) nouns is quite large in contrast. To account for their semantics, formal approaches have taken two main paths: one based on mereology following Link [6,7] and the other based on plural quantification following Boolos [8]. The latter develops plural logics intended to avoid the commitment to the existence of referents for plurals and group nouns on top of the referents of their members [9]. In this paper we aim to develop a view within the traditional firstorder framework, especially to make the proposal readily available to existing ontological systems. Moreover, we favour ontological precision over parsimony when both are inconciliable and so are prepared to adopt multiplicativism [10] when necessary. We will therefore rather take inspiration from mereological approaches.

A main issue in the literature on plurals is how to account for predication over plural nouns (see among many others $[6,9,11,12]$ ). Consider for instance plural noun phrases like 'the students' or 'Alice and Bob'. These refer to several things at once, i.e., to what is often called pluralities. Sometimes, one may reduce predication on plurals to independent claims for each element in the plurality: 'Alice and Bob are students' boils down to 
'Alice is a student' and 'Bob is a student', this is called distributivity. However, distributivity is blocked in expressions involving collective predicates like 'the students collaborate in the project', 'the cards are scattered on the floor' and 'all the workers met in the cafeteria'. Formally speaking, such examples of non-distributivity motivate the introduction of pluralities in the domain of quantification (or the use of plural quantifiers).

Plurals and singular group nouns are often accounted for without distinction in this literature, especially in the plural logics approach. Indeed, collective predicates apply to group nouns as well, viz. 'the deck (of cards) is scattered on the floor' and 'the committee met in the cafeteria'. Yet, some work in the mereology-based literature on group nouns [13] emphasised the need for distinguishing between the referents of group nouns and of plurals, that is, between collectives and pluralities. In contrast to pluralities, collectives manifest some sort of unity. It is not enough to have a plurality of musicians to have an orchestra: they need to play together or be bound to do so. Not any plurality of trees form a forest, they need to satisfy spatial constraints (and perhaps manifest ecological interrelationships). In addition, group nouns (and so kinds of collectives) usually convey the kind that characterises the collective's members: an orchestra is a collective of musicians, a forest a collective of trees, and one can go on with pairs like army-soldiers, herd-animals, deck-cards. Collectives thus display a homogeneity among members that plurals given in extension (with the conjunction 'and') as in 'Alice and her cat' do not possess. We will then distinguish three sorts of objects: pluralities, collectives, and composites, focusing on their internal structure and on the relationships between them.

Barker [13], expanding Link's proposal [6], showed how collectives are related to pluralities by some sort of constitution. For instance, if Alice and Bob are the members of a duet, the duet (a collective) is constituted by the plurality 'Alice and Bob'. Considering how constitution relates them throws further light on the differences between pluralities and collectives. The same plurality can constitute several collectives, even at the same time: an orchestra and a soccer team may have exactly the same members. Moreover, a plurality is directly bound to each of its members, and so it cannot change members without this changing the plurality itself. One can even introduce pluralities of objects existing at disjoint periods, as with 'Bach and Mozart'. In contrast, a collective can change members and so may be constituted at different times by different pluralities (of objects all existing at those times). Finally, while we may have collectives of collectives (e.g., federations of sport clubs), pluralities of pluralities are usually rejected (but see [14]), one reason why the literature on plurals embraced mereology rather than set theory. We also have pluralities of collectives: two orchestras are neither simply a plurality of musicians nor a larger single orchestra, just as 'two pairs of shoes' and 'four shoes' mean different things, a pair of shoes having its own unity.

The literature on constitution [15] is more concerned with composites than with collectives, and focused on what distinguishes composites from amounts of matter, often calling for notions such as form or function. Differences between composites and collectives are rarely addressed. The formal semantics literature above is also largely focused on comparing plurals and group nouns with mass nouns such as 'water', 'sand' or 'furniture' and little is said on how plurals or group nouns compare with count nouns referring to composites. We thus review here additional intuitions taken from various works that we take as starting point for developing our proposal.

Each composite has parts, that we will call components. Just as above with collectives, a composite differs from the plurality of its components. The classical literature on 
constitution argues at length that different pluralities of components may form a composite (say, my car) at different times, since some of its components (say, a tire) can change across time. Moreover, my car can be dismantled. The plurality of its components, scattered, does survive, while there would be no car there. So, as for collectives, we hold that there is a sense in which the composite is constituted by the plurality of its components.

Regarding the difference between composites and collectives, we know that membership is not transitive $[16,17,18]$, while componenthood is (albeit with some caveats $[16,19])$. Moreover, as we saw, the members of a collective are associated with a kind, thus in principle one can unambiguously count such members, let them be the musicians in an orchestra, the trees in a forest or the cards in a deck. On the contrary, trying to count the components of a composite may lead to several answers as it might be unclear what to count: the number of components of a lawn mower depends on the choice of a decomposition method (functional, structural, topological) and of the adopted level of granularity. A formal model able to capture the distinctions between pluralities, collectives and composites could prove helpful in representing information in such diverse scenarios as industrial plants, museums, galleries of art, systems of mechanical artefacts etc.

These concepts may also play an important role in the domain of social reality. The literature on metaphysics of social groups primarily addressed two questions: what kind of relationship holds between a social group and its members? What kind of entities (sums of individuals, sets of individuals, roles, etc.) is this relation connecting with social groups? Concerning the first question, there are in general two types of approaches, the former supporting some sort of mereological relation (for instance [20,21]) and the latter some sort of constitution relation ([22,23,24,25,26,27]). In the '80s Ruben [28,29] noted that there are three main properties of group membership that a metaphysical theory should represent: the persistence of groups through changes of their members; the existence of co-extensional but numerically distinct groups; and the non-transitivity of the relation between groups and members (properties already examined above). Concerning the second question, the view of groups as artefacts or organisms has been used as metaphor when addressing particular cases. But, as far as we know, the distinction of kinds of groups in terms of collectives and composites has been overlooked so far.

We propose a formal representation that captures the results of an analysis of the notions of plurality, collective and composite, and to test and apply it to the case of social groups and organisations. Although we illustrate our work on this specific application domain, we aim at generality, considering all sorts of objects, be they artefacts or natural objects, be they agentive or not. This will allow us to focus on the intrinsic structure of collectives and composites, and to refrain from calling for extrinsic properties such as function and agency that have been very often emphasised when characterising the relationships between a component and a composite or between a member and a collective. Although some argue in favour of a notion of function encompassing both artefacts and biological entities [30], we would not defend a function-based approach to composites as it would exclude from the start entities like mountains composed of peaks and valleys. Similarly, although many collectives are actually group agents, founding collectives on agency would exclude from the start forests and decks of cards.

The paper is organised as follows. Sect. 2 presents Link's proposal [6] as expanded by Barker [13], where collectives are related to pluralities by some sort of constitution, a starting point for our account. This general idea is sketched in Sect. 3 and is formally characterised in Sect. 4. Sect. 5 illustrates our account in the domain of social reality. 


\section{Pluralities and collectives in Link's and Barker's proposals}

In 1983 Link proposed a formal account ([6], reprinted in [7]) of collective predication, as in 'The children gather around their teachers', in analogy to predication involving mass nouns, like 'The water gathers in big pools.' Link's formal theory is rich and articulated. For what concerns us in this paper, we need only a fragment of his approach as presented below (symbols and terminology are our own), and as enhanced by Barker [13].

Link assumes the existence of two sets of entities, the set $O$ of (material) objects and the set $A$ of amounts of matter (with $A \subseteq O$ ), and two order relations, $\leq$ holding among objects and $(€$ holding among amounts of matter, with $(O, \leq)$ and $(A, \ominus)$ being join-semilattices. In a mereological perspective, join-semilattices can be characterised by imposing on $\leq$ and $\ominus$ the axioms of classical extensional mereology with unrestricted sum operators, see [31]. In Link's approach, $\leq$ (but not necessarily $(\diamond)$ ) is atomic and $\leq$-atoms are taken as referents of singular nouns, while non- $\leq$-atomic objects, that Link calls plural objects - our pluralities, are taken as referents of plural nouns. Link assumes that all amounts of matter are $\leq$-atoms.

Link adds a constitution function $h$ relating any object to the amount of matter making it up, where $h$ restricted to amounts of matter is identity, and with $h$ preserving order structures, i.e, mapping $\leq$ onto $\diamond$. Such a coupled double mereological structure enables Link to account for a significant number of linguistic phenomena involving plurals and mass nouns, clarifying their similarities and their differences.

Link makes explicit that collectives are to be distinguished from the pluralities of their members, collectives being $\leq$-atoms, and Barker [13] further enhances his proposal by adding a second constitution function $f$ over objects to relate collectives (called groups by Barker) to the pluralities making them up.

\section{Our approach in a nutshell}

Our account of pluralities, collectives, and composites formalised as a first-order theory in Sect. 4 builds on Link's and Barker's work, exploiting an atomic classical mereology to characterise pluralities. We will rephrase the constitution function $f$ between collectives and pluralities as a temporalised relation of constitution $\triangleleft$, since, as seen in Sect. 2, several arguments classically involved in constitution studies and based on change across time are used to distinguish these sorts of entities.

Since we are also interested here in social entities which do not have a clear (material) substrate, a second move is concerned with avoiding the commitment to a substrate when characterising the notions of plurality and collective. Note that this move does not prevent the integration of our proposal with foundational ontologies exploiting substrates (for instance, DOLCE [32] uses a constitution relation in the very same spirit of Link's function $h$ ). We leave such integration as future work but we point out in the following a possible enhancement of the axiomatisation based on the existence of a substrate; see the discussion on axiom (a11) below.

Finally, we introduce a second temporalised relation of constitution $\prec$ that links the composites to the pluralities of their components. As for collectives and pluralities, our characterisation of composites does not rely on the existence of a (material) substrate.

The strategy adopted, fully described in Sect. 4, is to account for the diversity of parthood relations on the basis of a diversity of constitution relations (namely $\triangleleft$ and $\prec$ ), 
exploiting a single mereological relation $\leq$. Importantly, $\leq$ is not meant to be a general parhood relation further specialised into a variety of parthood relations, as often suggested [16]. Rather, the atomic mereology built on $\leq$ is only aimed to model pluralities as finite sums of objects without assuming any unity or temporal constraint - in a spirit close to Lesniewski's efforts to eschew sets. The constitution relations, on the other hand, need to be temporalised to account for collective and composite changes across time. As required by Link, it is necessary that $\leq$ be atomic. Any entity considered as being singular, i.e., as having a unity, in particular any composite or collective, will be a $\leq$-atom, allowing it to be part of pluralities. Since $\leq$ is not a general parthood relation, being a $\leq$-atom only entails not being a plurality, and doesn't entail having no "parts" in a general sense of part.

\section{The formal account}

We consider two kinds of entities, namely objects (OB) like forests, cars, persons, etc. and times (TM), the class of instants. The framework can be extended to the case of events and temporal intervals, but for simplicity we focus here on objects and instants. To represent the presence (existence) of objects in time we introduce the binary predicate $\varepsilon$, with the formula $\varepsilon_{t} x$ standing for "the object $x$ is present at time $t$ ", (a1). ${ }^{1}$ We assume that any object is present at some time (a2).

$$
\begin{aligned}
& \text { a1 } \varepsilon_{t} x \rightarrow \mathrm{OB} x \wedge \mathrm{TM} t \\
& \text { a2 } \mathrm{OB} x \rightarrow \exists t\left(\varepsilon_{t} x\right)
\end{aligned}
$$

To describe pluralities we consider an atemporal mereological relation $\leq$ defined on objects (a3). Following standard practice in mereology, we require that the whole exists whenever at least one of its parts exists (a4). We assume in (a5) that $\leq$ satisfies the axioms of classical atomic ${ }^{2}$ extensional mereology [31] over objects, closed under unrestricted binary sum $(+)$ and, for objects that partially overlap, under binary difference $(-)$, see our implementation referred to in footnote 10 for the details.

$$
\begin{aligned}
& \text { a3 } x \leq y \rightarrow \text { ОВ } x \wedge \text { ОВ } y \\
& \text { a4 } x \leq y \wedge \varepsilon_{t} x \rightarrow \varepsilon_{t} y \\
& \text { a5 the axioms of closed atomic extensional mereology hold on } \leq,+ \text { and }- \text { over } \mathrm{OB}
\end{aligned}
$$

Definitions (d1) and (d2) standardly state that an atom is an object without proper parts, and that an atomic part is a part which is also an atom. We take pluralities to be non-atomic objects, that is, sums of two or more atomic objects. ${ }^{3}$ Pluralities may be present also when just some of their atoms are. The notion of wholly present is introduced in (d3) with the formula $\varepsilon_{t}^{\mathrm{w}} x$ standing for "the object $x$ is wholly present at time $t$ ", i.e., all the parts of $x$ are present at $t$. Note that some pluralities are never wholly present like the sum of temporally disjoint atoms, as with 'Bach and Mozart'.

$$
\text { d1 } \alpha x \triangleq \neg \exists y(y \leq x \wedge y \neq x)
$$

\footnotetext{
${ }^{1}$ To improve the reading of formulas, times are noted as subscripts.

${ }^{2}$ We adopt the atomicity axiom $\forall x\left(\mathrm{OB} x \rightarrow \exists y\left(y \leq_{\alpha} x\right)\right)$, using (d2). With strong supplementation (OB $x \wedge$ ов $y \wedge \neg(x \leq y) \rightarrow \exists z(z \leq x \wedge \neg \exists v(v \leq z \wedge v \leq y))))$ two entities having the same atomic parts are identical.

${ }^{3}$ As explained above, atoms correspond to singular objects as opposed to pluralities; this doesn't mean that an atom cannot have components or members, notions that are not captured by $\leq$.
} 
d2 $x \leq \alpha y \triangleq x \leq y \wedge \alpha x$

d3 $\varepsilon_{t}^{\mathrm{W}} x \triangleq \forall y\left(y \leq x \rightarrow \varepsilon_{t} y\right)$ (atomic part)

( $x$ is wholly present, wholly exists, at $t$ )

In the following we pursue the idea of grounding the distinction between collectives and composites using two temporally qualified constitution primitives (a6):

$-x \triangleleft_{t} y$ is meant to hold between a collective and a (wholly present) plurality;

$-x \prec_{t} y$ is meant to hold between a composite and a (wholly present) plurality.

The plurality constituting the object may change from time to time, however, if an object is $\triangleleft$ - or $\prec$-constituted at some time, then it is so for all its life, see (a7) and (a8).

$$
\begin{aligned}
& \text { a6 }\left(x \prec_{t} y \vee x \triangleleft_{t} y\right) \rightarrow \varepsilon_{t} x \wedge \varepsilon_{t}^{\mathrm{W}} y \wedge \alpha x \wedge \neg \alpha y \\
& \text { a7 } \varepsilon_{t} x \wedge \varepsilon_{t^{\prime}} x \rightarrow\left(\exists y\left(x \triangleleft_{t} y\right) \leftrightarrow \exists y^{\prime}\left(x \triangleleft_{t^{\prime}} y^{\prime}\right)\right) \\
& \text { a8 } \varepsilon_{t} x \wedge \varepsilon_{t^{\prime}} x \rightarrow\left(\exists y\left(x \prec_{t} y\right) \leftrightarrow \exists y^{\prime}\left(x \prec_{t^{\prime}} y^{\prime}\right)\right)
\end{aligned}
$$

We do not enforce unicity on these relations: the same plurality can constitute different collectives as well as different composites, and it can constitute both collectives and composites. For instance, consider a deck of cards, a collective, which has been arranged into a tower, a composite. Each card is at the same time a member of the deck and a component of the tower, so the plurality of cards $\triangleleft$ - and $\prec$-constitutes the deck and the tower, respectively. Furthermore, composites can be members of collectives and collectives can be components of composites.

Example 1 (Modeling a forest as collective and a larch as composite). To model a forest $w$ as a collective of larches (say, just $l_{1}$ and $l_{2}$ for simplicity), we can write the formula: $w \triangleleft_{t}\left(l_{1}+l_{2}\right)$. To model each larch as a composite of crown and trunk (say, $c r_{i}$ and $t r_{i}$ ), we can write: $l_{1} \prec_{t}\left(c r_{1}+t r_{1}\right) \wedge l_{2} \prec_{t}\left(c r_{2}+t r_{2}\right)$, see Fig. 1 (solid edges represent $\leq$ and all bottom objects are $\leq$-atomic; we omit many sums for clarity).

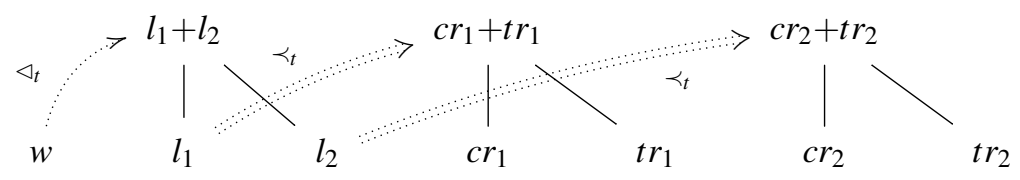

Figure 1. Modeling a forest as a collective and a larch tree as a composite.

Example 2 (Modeling a larch as composite with the crown component having foliage as a collective component). To model a larch $l$ as a composite of trunk $t r$, and crown $\mathrm{cr}$ we write $l \prec_{t}(t r+c r)$, to model the crown $c r$ as a composite with two branches $b_{1}, b_{2}$ and foliage $f$ we write $c r \prec_{t}\left(b_{1}+b_{2}+f\right)$, and for foliage $f$ being a collective of leaves, say, $l v_{1}$ and $l v_{2}$, we write $f \triangleleft_{t}\left(l v_{1}+l v_{2}\right)$, see Fig. 2 .

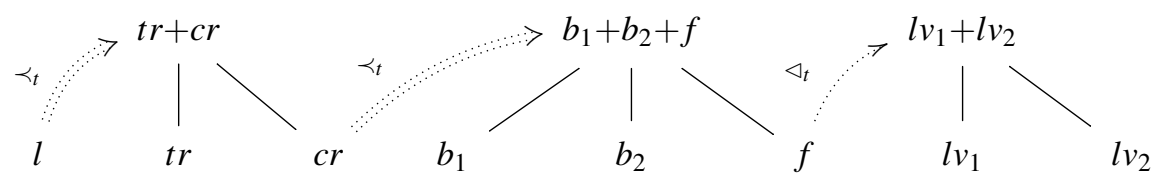

Figure 2. Modeling a larch as a composite with a component involving a collective. 
The notions of being collective and being member of are defined in terms of $\triangleleft$, see (d4) and (d6), while the notions of being composite and being component of are defined in terms of $\prec$, see (d5) and (d7). From the definitions, members and components must be atomic objects, in line with Link's proposal where all singular objects are atoms. Note that collectives and composites are not assumed to be disjoint, i.e., it is possible for an object to be both $\triangleleft$ - and $\prec$-constituted, a hypothesis explored at the end of this section.

$$
\begin{aligned}
& \text { d4 } \mathrm{CL} x \triangleq \forall t\left(\varepsilon_{t} x \rightarrow \exists y\left(x \triangleleft_{t} y\right)\right) \\
& \text { d5 } \mathrm{CM} x \triangleq \forall t\left(\varepsilon_{t} x \rightarrow \exists y\left(x \prec_{t} y\right)\right) \\
& \text { d6 } x \operatorname{memb}_{t} y \triangleq \exists z\left(y \triangleleft_{t} z \wedge x \leq_{\alpha} z\right) \\
& \text { d7 } x \operatorname{comp}_{t} y \triangleq \exists z\left(y \prec_{t} z \wedge x \leq_{\alpha} z\right)
\end{aligned}
$$

(being a collective)

(being a composite)

(being member of)

The adoption of the above definitions makes it crucially important to distinguish $\triangleleft$ from $\prec$. Up to this point, they have the same characteristics, cf. (a6)-(a8). To formally differentiate the two constitution primitives, a first possibility is to identify a difference in the way pluralities are structured to constitute collectives vs. composites. As seen in Sect. 1 , the literature, e.g. [4,5], considers that collectives have a uniform structure, while assemblies or functional complexes have a heterogeneous one. Some authors [33,34] further elaborate this idea by claiming that in a collective all the members play the same role, while components usually play a variety of different roles in a functional complex.

The notion of functional complex seems more restrictive than our notion of composite. For example, it seems not quite natural to consider the functional aspects of composites like mountains or molecules as fundamental, and even awkward to ask what roles could be involved in their structures. Second, this move requires us to formally characterise the notions of structure and role. Following Fine [35], a structure could be represented by means of a relation holding among the members or components. Collectives and composites could then be reduced to variable embodiments, i.e., entities that at any time $t$ at which they are present are constituted by a rigid embodiment, i.e., a sort of compound of the objects, say, $a_{1}, \ldots, a_{n}$, and the relation $R$ connecting them at $t$, shortly written $\left[a_{1}, \ldots, a_{n} / R\right]$. For instance, suppose that a car changes its engine from $t_{1}$ to $t_{2}$ and, for simplicity, that cars have just two components, namely an engine and a frame. According to Fine, the rigid embodiment $\left[e_{1}, f / R\right]$ that constitutes the car at $t_{1}$ is different from the rigid embodiment $\left[e_{2}, f / R\right]$ that constitutes the car at $t_{2}$, where $e_{1}$ and $e_{2}$ are two different engines and $f$ is the frame. Note that both the rigid embodiments $\left[e_{1}, f / R\right]$ and $\left[e_{2}, f / R\right]$ and the pluralities $e_{1}+f$ and $e_{2}+f$ are compositionally static, but $e_{1}+f$ is wholly present whenever all its atoms are present, while $\left[e_{1}, f / R\right]$ requires the holding (at $\left.t_{1}\right)$ of $R\left(e_{1}, f\right)$ in addition to the existence of $e_{1}$ and $f$.

The introduction of variable embodiments has some drawbacks. First, it requires an additional kind of entity, namely, the rigid embodiments. The nature of rigid embodiments seems quite close to that of states in [36], but Fine, more recently, prefers to liken their ontological status to that of qua-entities [37]. Second, one could assume that variable embodiments are always constituted by rigid embodiments grounded on the same relation $R$. Even though this assumption seems in line with Rector and colleagues' approach, where composites are always composed by a determinate number of parts [33], it has been considered too restrictive by Jansen and Schulz [3], it does not apply to collectives that can lose or acquire members, and it is not endorsed by Fine himself. A variable embodiment can then be constituted, at different times, by rigid embodiments that are grounded on different relations (possibly with different arities). Therefore, by assuming 
that a given type of collectives or composites is associated with one structure, this structure cannot in general coincide with a single relation $R$, it should consist of the variety of relations, each one grounding a rigid embodiment, constituting the overall variable embodiment at some point in time. ${ }^{4}$ One then should look for (meta-)criteria to characterise which kind of relations can be associated to a variable embodiment of a given type. Fine does not address this point (see [39] for additional criticisms).

As shown by Uzquiano [40], the introduction of pluralities avoids the commitment to rigid embodiments and it may alleviate the previous problem because the same property can apply to pluralities collecting different numbers of objects. In our framework-in line with the structural-constitution view introduced by Harris for group agents [25] given finite sets $\mathscr{T}_{\mathrm{CL}}$ and $\mathscr{T}_{\mathrm{CM}}$ of unary predicates that represent types of, respectively, collectives and composites, one can introduce sufficient (and necessary) conditions for the existence of collectives or composites of a given type. More precisely, for each $\mathrm{P} \in$ $\mathscr{T}_{\mathrm{CL}}$ an axiom with form (f1) can be introduced to ensure that for each wholly present plurality $x$ satisfying $\mathrm{F}$ there exists a collective of type P constituted by $x$ that during its whole life is constituted by pluralities satisfying the property $F$, which is assumed to be flexible enough to allow changes in the number and in the configuration of the members. For instance, for forests, F should ensure that all the trees are spatially interconnected in a possibly quite general way (e.g., trees' neighbour distance is below a threshold and the plurality is maximal with respect to the distance criterion). Analogously, for each $\mathrm{Q} \in \mathscr{T}_{\mathrm{CM}}$ an axiom of the form (f2) can be added. ${ }^{5}$ The approach can be strengthened by adding necessary conditions following (f3) and (f4).

$$
\begin{aligned}
& \text { f1 } \mathrm{F}_{t} x \wedge \neg \alpha x \wedge \varepsilon_{t}^{\mathrm{W}} x \rightarrow \exists y\left(\mathrm{P} y \wedge y \triangleleft_{t} x \wedge \forall t^{\prime}\left(\varepsilon_{t^{\prime}} y \rightarrow \exists x^{\prime}\left(y \triangleleft_{t^{\prime}} x^{\prime} \wedge \mathrm{F}_{t^{\prime}} x^{\prime}\right)\right)\right) \\
& \text { f2 } \mathrm{G}_{t} x \wedge \neg \alpha x \wedge \varepsilon_{t}^{\mathrm{W}} x \rightarrow \exists y\left(\mathrm{Q} y \wedge y \prec_{t} x \wedge \forall t^{\prime}\left(\varepsilon_{t^{\prime}} y \rightarrow \exists x^{\prime}\left(y \prec_{t^{\prime}} x^{\prime} \wedge \mathrm{G}_{t^{\prime}} x^{\prime}\right)\right)\right) \\
& \text { f3 } \mathrm{P} y \rightarrow \forall t\left(\varepsilon_{t} y \rightarrow \exists x\left(y \triangleleft_{t} x \wedge \mathrm{F}_{t} x\right)\right) \\
& \text { f4 Q } y \rightarrow \forall t\left(\varepsilon_{t} y \rightarrow \exists x\left(y \prec_{t} x \wedge \mathrm{G}_{t} x\right)\right)
\end{aligned}
$$

Axioms with forms (f1)-(f4) still do not distinguish between $\triangleleft$ and $\prec$ : all the types in $\mathscr{T}_{\mathrm{CL}}$ and $\mathscr{T}_{\mathrm{CM}}$ have associated properties representing sufficient (and necessary) conditions. Are there differences between the nature of the properties $F$ associated with the types of collectives and the nature of the properties $\mathrm{G}$ associated with the types of composites? Wilson [41] proposes to separate compositional constitution from ampliative constitution on the basis of the structure they rely upon. A structure is intrinsic when it holds only in virtue of how the constituents are interlinked, e.g., the bonds between the $\mathrm{H}$ and $\mathrm{O}$ atoms in a $\mathrm{H}_{2} \mathrm{O}$ molecule. A structure is extrinsic when it holds because of some relations in which also non-constituent entities intervene, e.g., artefacts are usually defined also referring to intended capabilities and uses. Wilson's distinction seems however orthogonal to the one between $\triangleleft$ and $\prec$. Collectives like forests and composites like molecules seem both grounded on intrinsic structures while collectives like organisations and composites like artefacts on extrinsic structures. It seems then difficult to differentiate the nature of the "structures" of collectives vs. composites. Jansen and Schulz [3] re-

\footnotetext{
${ }^{4}$ Unless one considers polyadic or multigrade relations, see [38].

${ }^{5}$ Note that, if two distinct collectives of type P constituted by the same plurality exist (something consistent with our framework), an axiom with form (f1) guarantees the existence of just one collective. This could be the case, for instance, with two distinct societies of the same legal type $\mathrm{P}$ having exactly the same members and so the same constituting plurality instantiating F once. The rigid embodiment approach [35] could make the difference in case the members fill different arguments (roles) in some fine-grained enough relation $R$.
} 
port that the BioTop Ontology ${ }^{6}$ assumes that the components of composites are spatially self-connected, while the members of collectives are spatially scattered. However, they recognise that this constraint appears too strict even within the field of bio-medicine.

A promising alternative is to focus on the intuitive uniformity among the members of a collective; indeed, as pointed in Sect. 1, giving a collective one tends to specify the type of their members. This is not the case of composites that can include quite heterogeneous components. Following this intuition, Rector and colleagues [33] as well as Galton [42] assume that all the members of a collective are of a specified type. In our framework, via an axiom with form (f5), one can associate to each $\mathrm{P} \in \mathscr{T}_{\mathrm{CL}}$ a type $\mathrm{T}$ applying to members. Galton does not require the type to be unique, but requires that the types characterising the members of a collective are closed under subsumption. Axioms of the form (f5) can then be introduced only for the minimal type (w.r.t. subsumption). ${ }^{7}$

f5 $\mathrm{P} y \wedge x \mathrm{memb}_{t} y \rightarrow \mathrm{T} x$

Unfortunately, this strategy presents problems similar to those met discussing structures of collectives and composites. Indeed, nothing prevents axioms with form (f5) from being applied to composites. For instance, all the components of a car are of type being an artefact. One can object that there is an important difference between types like being a tree and being an artefact: the first seems to capture quite closely the very nature of the objects one aims to classify. The latter, at least informally, seems to be characterised by much weaker conditions. In an ontological hierarchy, one expects to find being a tree at a lower level than being an artefact. However, it remains unclear whether such a difference on types could be drawn only on the basis of the level of ontological generality or whether this difference should be characterised in an alternative way.

Therefore, axioms with form (f1)-(f5) are still quite weak to clearly separate collectives from composites. We now introduce two further intuitions to differentiate $\triangleleft$ and $\prec$. First, we observe that collectives decompose into members in a unique way. The members of a forest are trees, the members of a crowd are persons. In contrast, in composite objects one often has a choice of possible decompositions. For instance, (the body of) a person may be decomposed into organs or, alternatively, into body parts like arms, legs, trunk, head and the like. In other words, to determine the components of a compositebut not the members of a collective-one needs to make some decomposition criterion explicit. This idea is also in line with the approach followed in [3], where components are always relative to a certain "partition"- $\mathrm{a}$ "(mechanical or cognitive) act or process of dividing something into parts" $[3, \mathrm{p} .5]$ —of a composite. ${ }^{8}$

We enforce the unique decomposition of collectives by means of (a9). Composites are not constrained by an anologous axiom, i.e., it is possible to have $x \prec_{t} y \wedge x \prec_{t} z \wedge y \neq z$. This also means that composites behave differently from variable embodiments: at every time at which a variable embodiment exists it has a unique rigid embodiment, thus synchronic "decompositions" into different components are banned in Fine's theory.

a9 $x \triangleleft_{t} y \wedge x \triangleleft_{t} z \rightarrow y=z$

\footnotetext{
${ }^{6}$ http://purl.org/biotop/

${ }^{7}$ Galton assumes that if members of a collective are both of type $\mathrm{T}$ and $\mathrm{T}^{\prime}$, they also are of type $\mathrm{T}^{\prime \prime}$, where $\mathrm{T}^{\prime \prime}$ is subsumed by $\mathrm{T}$ and $\mathrm{T}^{\prime}$, cf. axiom COLOF4 [42, p.16], to guarantee the existence of a unique minimal type.

${ }^{8}$ In [3] the components of a partition form a collective, while we here assume that they form a plurality.
} 
The second intuition concerns recursive decomposition. Roughly speaking, it is possible to recursively decompose composites but not collectives. This is what the transitivity of relationships is about. As seen in Sect. 1, the members of the members of a collective are not members of the collective itself: memb is not transitive. For instance, the members of the countries that are members of UN, e.g., the federal states of USA are not members of UN. On the other hand, the components of a component of a composite are themselves components of the composite; consider the case of the branches of the crown of a tree in Example 2 above, the screws of the engine of a car, or the handle of the door of a house (although many would claim the handle is not a functional part of the house [19]). Similarly, the members of a collective which is a component of a composite are also components, see the case of the leaves of the foliage of a tree in Example 2. Rector and colleagues [33] also contrast a non-transitive granular parthood holding on collectives and a transitive determinate parthood holding on composites.

We formalise the recursive decomposition of composites in (a10). Importantly, this does not have a correspondent for relation $\triangleleft$. We need to ensure that alternative decompositions wholly "cover" the same composite. However, in the case of decompositions recursively ending up in atoms of different types, as in the previous example of body parts vs. organs, it is necessary to rely on some relationship between them, for instance using a common (material or spatial) substrate. Leaving such an extension for future work, (a11) simply excludes that one of the decompositions is a proper part of the other.

$$
\begin{aligned}
& \text { a10 } x \prec_{t} y \wedge a \leq_{\alpha} y \wedge\left(a \prec_{t} z \vee a \triangleleft_{t} z\right) \rightarrow x \prec_{t}((y-a)+z) \\
& \text { a11 } x \prec_{t} y \wedge x \prec_{t} z \rightarrow \neg(y \leq z \wedge y \neq z)
\end{aligned}
$$

Since $x \prec_{t} y \wedge y \prec_{t} z \rightarrow x \prec_{t} z$ is trivially true (likewise with $\triangleleft$ ) because the antecedent never holds ( $y$ cannot be both a plurality and an atom), it is more relevant to investigate the transitivity and other properties of comp and memb. The irreflexivity of memb and comp cannot yet be proven because nothing prevents an object from being an atomic part of the plurality that constitutes it, i.e., one of its own constituents. These unintended models are directly ruled out by (a12) — where the overlap relation $\gamma$ is defined in (d8)—see (t1).

$$
\begin{array}{rl}
\mathbf{d 8} & x \curlyvee y \triangleq \exists z(z \leq x \wedge z \leq y) \\
\mathbf{a 1 2} & \left(x \triangleleft_{t} y \vee x \prec_{t} y\right) \rightarrow \neg(x \curlyvee y) \\
\mathbf{t 1} & \neg\left(x \operatorname{memb}_{t} x\right) \wedge \neg\left(x \operatorname{comp}_{t} x\right)
\end{array}
$$

The transitivity of comp (t2) and the "mixed" transitivity of memb and comp (t3) follow from (d7) and (a10), therefore comp is also asymmetric (t4). Vice versa, as desired, memb is not transitive because there are no axioms that, given $\exists a\left(y \triangleleft_{t} a \wedge x \leq_{\alpha} a\right)$ and $\exists b\left(z \triangleleft_{t} b \wedge y \leq_{\alpha} b\right)$, ensure a link between $a$ and $b$ (by (a9), $b$ is the unique plurality that constitutes $z$ ). Even though the putative constraint (p1) ${ }^{9}$ would guarantee the antitransitivity of memb (p2), some examples bring evidence against it. For instance, consider an organisation $O$ that among its members accepts both persons and organisations. In this case, John could be a member of the University of Oxford, and both John and the University of Oxford be members of $O$ against (p2). Since the asymmetry of memb cannot yet be proven but is desirable, we impose it via (a13). Finally, a form of weak supplementation holds for both memb and comp, see (t5) and (t6).

\footnotetext{
${ }^{9}$ We label with $(\mathrm{p} x)$ constraints that we want to discuss but are not included in the theory.
} 
t2 $x \operatorname{comp}_{t} y \wedge y \operatorname{comp}_{t} z \rightarrow x \operatorname{comp}_{t} z$

Proof. From the hypothesis and (d7), $\exists a\left(y \prec_{t} a \wedge x \leq_{\alpha} a\right)$ and $\exists b\left(z \prec_{t} b \wedge y \leq_{\alpha} b\right)$, i.e., $\exists a b\left(z \prec_{t} b \wedge y \leq_{\alpha} b \wedge y \prec_{t} a\right)$. By (a10), the fact that $x \leq_{\alpha} a$, and the transitivity of $\leq$ it follows that $z \prec_{t}((b-y)+a) \wedge x \leq_{\alpha}((b-y)+a)$, i.e., $x \operatorname{comp}_{t} z$.

$\mathbf{t 3} x \mathrm{memb}_{t} y \wedge y \operatorname{comp}_{t} z \rightarrow x \operatorname{comp}_{t} z$

(see the proof of (t2))

t4 $x \operatorname{comp}_{t} y \rightarrow \neg\left(y \operatorname{comp}_{t} x\right)$

(directly from (t1) and (t2))

p1 $x \triangleleft_{t} y \wedge a \leq_{\alpha} y \wedge a \triangleleft_{t} z \rightarrow \neg(y \curlyvee z)$

p2 $x$ memb $_{t} y \wedge y$ memb $_{t} z \rightarrow \neg\left(x \operatorname{memb}_{t} z\right)$

a13 $x \operatorname{memb}_{t} y \rightarrow \neg\left(y \operatorname{memb}_{t} x\right)$

t5 $x \operatorname{memb}_{t} y \rightarrow \exists z\left(\neg(z \gamma x) \wedge z \operatorname{memb}_{t} y\right)$

(directly from (d1), (d2), (d6), (a5), (a6))

t6 $x \operatorname{comp}_{t} y \rightarrow \exists z\left(\neg(z \chi x) \wedge z \operatorname{comp}_{t} y\right)$

(directly from (d1), (d2), (d7), (a5), (a6))

We can introduce subcollective and subcomposite relations via (d9) and (d10). Both subcl and subcm are trivially reflexive (at a given time). However, given the fact that the same plurality can constitute different objects subcm and subcl are not antisymmetric, and while subcl is transitive ( $\mathrm{t} 7$ ), the fact that a composite can be decomposed in different ways rules out the transitivity of subcm.

$$
\begin{aligned}
\text { d9 } & x \text { subcl }_{t} y \triangleq \exists a b\left(x \triangleleft_{t} a \wedge y \triangleleft_{t} b \wedge a \leq b\right) \\
\text { d10 } & x \text { subcm }_{t} y \triangleq \exists a b\left(x \prec_{t} a \wedge y \prec_{t} b \wedge a \leq b\right) \\
\text { t7 } & x \text { subcl }_{t} y \wedge y \operatorname{subcl}_{t} z \rightarrow x \text { subcl }_{t} z
\end{aligned}
$$

Proof. From the hypothesis and (d9) we have $\exists a b\left(x \triangleleft_{t} a \wedge y \triangleleft_{t} b \wedge a \leq b\right)$ and $\exists c d\left(y \triangleleft_{t} c \wedge z \triangleleft_{t} d \wedge c \leq b\right)$. By (a9), we have $b=c$, and by the transitivity of $\leq$, $a \leq d$. Thus $\exists a d\left(x \triangleleft_{t} a \wedge z \triangleleft_{t} d \wedge a \leq d\right)$.

Note that $x \prec_{t}(y+z), y \prec_{t}(a+b)$, and $z \prec_{t}(c+d)$ imply $x \prec_{t}(a+b+c+d)$ by (a10). The other direction does not hold: $x \prec_{t}(a+b+c+d)$ does not imply the existence of objects $y$ and $z$ that are both subcomposites and components of $x$. One could try to resume Fine's approach [43] to model the difference between these two situations. In [43], Fine introduced a summation operator $\Sigma$ such that $\Sigma\left(\Sigma\left(x_{1}, x_{2}, \ldots\right), \Sigma\left(y_{1}, y_{2}, \ldots\right)\right)$ is different from $\Sigma\left(x_{1}, x_{2}, \ldots, y_{1}, y_{2}, \ldots\right)$. However, $\Sigma$ is not temporally qualified, therefore even though $x \prec_{t} \Sigma(\Sigma(a, b), \Sigma(c, d)), x \prec_{t} \Sigma(a, b, c, d)$ and the two sums are different, this cannot be used to provide the needed $y$ and $z$ (with $y \prec_{t} \Sigma(a, b)$ and $z \prec_{t} \Sigma(c, d)$ ) since in our framework $y$ and $z$ can change their components through time. Our framework is thus richer and requires more expressive operators.

As said, collectives and composites are not disjoint. This possibility is particularly interesting for social groups and organisations that can be described not only in terms of their members but also in terms of the variety of decompositions into departments or committees with specific roles within the organisation or group. In fact, approaches in which collectives and composites are disjoint and which insist on the homogeneity of collectives to conclude that organisations with differentiated roles such as a string trio (three musicians playing a violin, a viola and a cello) can be considered as a composite are unable to account for the fact that they have members, i.e., that members can be unambiguously counted (quite obvious for a trio), that some homogeneity is present since members are of a same type (musicians for a trio), and that no component of a member (e.g., Lea's foot) is relevant for the organisation. Sect. 5 discusses additional examples.

Additional constrains are needed for composites that are also collectives. First, we ensure that alternative decompositions "cover" the whole composite-collective, complet- 
ing (a11) with (a14), which guarantees that all the members also are components. Second, we make sure with (a15) that the composite and the collective views match up to the member's level, i.e., that all the components "larger" than a member are also subcollectives. By (t2), components of members, e.g., Lea's foot, still are components of composite-collectives like the string trio. So, to avoid considering such components, we finally introduce compcl (component of collective) as a (non-transitive) sub-relation of comp dedicated to compositive-collectives that stops at member's level, see (d11).

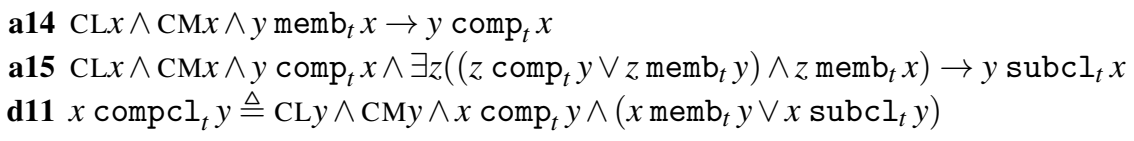

The proposed theory has been implemented and tested for consistency by means of theorem provers. ${ }^{10}$ More precisely, (a1)-(a15) is a consistent theory, and its extension with sample axioms of forms (f1)-(f5) has been proved consistent as well.

\section{An application: social groups}

We now analyse how social groups can be represented as collectives and/or composites in our account. Adopting this framework, we assume an anti-reductionist position on groups: both collectives and composites are constituted by and distinct from pluralities. So our approach stands on the side of the constitution-based ones [22,23,24,25,26,27]. This literature usually assumes the existence of properties keeping group members together. In most cases, such property is taken to be the structure, which some authors specify as functional structure. We take this to be similar to what we proposed with axioms (f1)-(f4) for collectives and composites. Moreover, some authors require all members to belong to a same type, e.g, being a person, a social entity or an agent. This is in line with (f5). Finally, as in all these theories based on constitution, we can account for groups surviving the change of their members and for different groups sharing their members.

Now, in this framework, should social groups be considered as collectives or as composites? Our position is that all social groups are collectives, but that only some of them are also composites. This choice is motivated by the fact that collectives but not composites have an associated member-type (f5), that (a9) leaves no ambiguity on what is a member of a collective, but that some groups, like composites, can have heterogeneous components and can be decomposed in alternative ways. We will thus talk of groups that are pure collectives and of those that are composite-collectives. Accounting for the double nature of the latter groups is made possible in our framework where collectives and composites are not disjoint, and where compcl is the relation of choice for them.

Fine [27] highlights that the structure of social groups can be more or less layered and articulated. In our framework the variety of the layered structures of social groups can be captured by chaining $\triangleleft$ - and $\prec$-constitutions. For example, we can have collectives that have as members other collectives, e.g., the European Union, which has member States (that, arguably, are in their turn collectives), collectives with both collectives and individuals (i.e., non-collective objects) as members, e.g., scientific associations which can have both individuals and institutions as members (in this case, the members or the components of the institutions are not necessarily members of the association), compos-

\footnotetext{
${ }^{10}$ See https://github.com/diporello/plurals/blob/master/ontology_of_plurals.p
} 
ites that have as components only collectives, only composites, collectives and individuals (composites or not), up to the most complex cases, like big companies, which can have as components individuals, e.g. the President, collective-composites, e.g. an executive board composed of subgroups like an education and a communication committee, and pure collectives, i.e., all the other members. It is important to stress here that while collectives directly provide the members, composites allow us to finely specify their structural decomposition. Suppose, for example, to have the following collectives: $c$ is the Milan football team (with 11 football players), $c_{1}$ the defense, $c_{2}$ the attack, $c_{3}$ is a musical trio (composed by 3 footballers), and $c_{4}$ a bridge club (composed by the remaining 8 footballers). Because the members of $c_{1-4}$ are among the ones of $c$, by (d9), $c_{1-4}$ are all subcollectives (but not members) of $c$. Our composite-collectives allow, via (a14) and (a15), to include among the components only some subcollectives, e.g., the Milan football team $c$ can be represented as a composite-collective with (in addition to the 11 footballers) $c_{1}$ and $c_{2}$, but not $c_{3}$ and $c_{4}$, as components. Composite social groups can then range from the Italian Parliament—which has as members the parliamentarians, but can be decomposed in different ways (e.g., in Chamber and Senate, as well as in parliamentarian groups) — to the aforesaid Milan club that indeed, differently from pure collectives, can be decomposed into defense and attack but also in alternative or more fine-grained ways, e.g., identifying goal-keepers, defenders, midfielders and strikers. ${ }^{11}$

Though most examples seem to rely on a notion of structure that is tightly connected with functionality or with power-responsibility relations-a fact that deeply influenced the social ontology literature, our approach allows to keep these two dimensions separate. Structure can in our framework rely on very different rationales. This seems to be a desirable feature for social groups, as it allows to account for the various ways in which a complex organisation can be synchronically organised. For instance, we could decide to decompose a composite social group, like a multinational company whose offices occupy four floors of a building, on the basis of the floor in which offices are located as well as on the basis of the organisational charter defining roles and responsibilities, etc.

Another relevant distinction in the case of social groups is that between mere groups and group agents; in [44] we have argued that what distinguishes them is that the latter have an established decision procedure, which allows them to act as a whole, while the former do not. Though collective agency is often discussed in connection with structure, in our approach the two are orthogonal dimensions. In fact, we could have non-agentive as well as agentive pure collectives (or groups and group agents) and both agentive and non agentive composite-collectives (or composite groups and composite group agents). An example of non-agentive pure collective is the collective of Starbucks bartenders (neither one of them taken individually nor they together can act on behalf of Starbucks), while an agentive pure collective is the Supreme Court of the United States. Parliaments and sport teams are agentive composite-collectives, while an example of non agentive composite-collective is a choir of an orchestra, which can be decomposed in sopranos, baritones, tenors and so on, but whose decisions (for instance which songs to sing) are taken by someone who's not a component of the choir. Something worth pointing out is that, in all cases, including social groups that have both members and subcollectives as components, the fact that the group is agentive or not is not a direct consequence of

\footnotetext{
${ }^{11} \mathrm{By}(\mathrm{a} 14)$ and (a15), the most fine-grained compcl-decomposition of a social group represented as a composite-collective is always given in terms of the members of the collective.
} 
its components being agentive or not, but it rather depends on the group having its own decisional procedure, allowing it to act as a whole.

\section{Conclusions}

This paper presents the core of a formal theory for representing the notions of plurality, collective and composite. The proposed theory is the result of an analysis of various formal approaches in the literature and of their drawbacks. The focus has been on the intrinsic structure of collectives and composites brought to the light by the use of two different relations of constitution. In particular, we were interested in developing a framework applicable to social reality, where a theory of this kind is definitely needed. However, the scope of the theory is more general, spanning natural objects, artefacts, and agentive/non-agentive entities. Our aims for the future are on the one hand to enrich the theory with functionalities and roles and to further develop its application to social reality. On the other hand, we plan its integration into some foundational ontology, mapping $\leq$ (with appropriate temporal constraints on the relata), comp and memb into a general temporalised parthood on objects, and introducing in the picture the amounts of matter constituting material objects, as Link did.

Acknowledgements We are grateful to Adrien Barton, Kit Fine, and four anonymous reviewers for helpful comments.

\section{References}

[1] Bottazzi E, Catenacci C, Gangemi A, Lehmann J. From collective intentionality to intentional collectives: An ontological perspective. Cognitive Systems Research. 2006;7(2-3):192-208.

[2] Galton A, Wood Z. Extensional and intensional collectives and the de re/de dicto distinction. Applied Ontology. 2016;11(3):205-226.

[3] Jansen L, Schulz S; Springer. Grains, components and mixtures in biomedical ontologies. Journal of Biomedical Semantics. 2011;2(Suppl 4):S2.

[4] Winston M, Chaffin R, Herrmann D. A taxonomy of part-whole relations. Cognitive Science. 1987;11(4):417-444.

[5] Gerstl P, Pribbenow S. Midwinters, end games, and body parts: a classification of part-whole relations. International Journal of Human-Computer Studies. 1995;43(5-6):865-889.

[6] Link G. The logical analysis of plurals and mass terms: A lattice theoretical approach. In: Bäuerle R, Schwarze C, von Stechow A, editors. Meaning, Use and the Interpretation of Language. de Gruyter; 1983. p. 302-323.

[7] Link G. Algebraic Semantics in Language and Philosophy. Lecture Notes. CSLI; 1998.

[8] Boolos G. To Be Is To Be a Value of a Variable (or to Be Some Values of Some Variables). The Journal of Philosophy. 1984;81(8):430-449.

[9] Linnebo Ø. Plural Quantification. In: Zalta E, editor. The Stanford Encyclopedia of Philosophy. Summer 2017 ed. Stanford University; 2017.

[10] Vieu L, Borgo S, Masolo C. Artefacts and Roles: Modelling Strategies in a Multiplicative Ontology. In: Eschenbach C, Gruninger M, editors. Proceedings of Fifth International Conference on Formal Ontology and Information Systems (FOIS 2008). IOS Press; 2008. p. 121-134.

[11] Moltmann F. Parts and Wholes in Semantics. Oxford University Press; 1997.

[12] Winter Y. Atom predicates and set predicates: Towards a general theory of plural quantification. In: Semantics and Linguistic Theory. vol. 8. Cornell University; 1998. p. 249-266.

[13] Barker C. Group terms in English: Representing groups as atoms. Journal of Semantics. 1992;9(1):69_ 93. 
[14] Linnebo Ø, Nicolas D. Superplurals in English. Analysis. 2008;68(3):609-681.

[15] Wasserman R. Material Constitution. In: Zalta E, editor. The Stanford Encyclopedia of Philosophy. Fall 2018 ed. Stanford University; 2018.

[16] Varzi A. A note on the Transitivity of Parthood. Applied Ontology. 2006;1(2):141-146.

[17] Vieu L, Aurnague M. Part-of Relations, Functionality and Dependence. In: Aurnague M, Hickmann M, Vieu L, editors. The Categorization of Spatial Entities in Language and Cognition. John Benjamins; 2007. p. 307-336.

[18] Keet M, Artale A. Representing and reasoning over a taxonomy of part-whole relations. Applied Ontology. 2008;3(1-2):91-110.

[19] Vieu L. On the transitivity of functional parthood. Applied Ontology. 2006;1(2):147-155.

[20] Hawley K. Social Mereology. Journal of the American Philosophical Association. 2017;3(4):395-411.

[21] Strohmaier D. Group Membership and Parthood. Journal of Social Ontology. 2019;4(2):121-135.

[22] Sheehy P. The Reality of Social Groups. Routledge; 2006.

[23] Epstein B. The Ant Trap: Rebuilding the Foundations of the Social Sciences. Oxford University Press; 2015.

[24] Thomasson A. The Ontology of Social Groups. Synthese. 2019;196(12):4829-4845.

[25] Harris K. How Individuals Constitute Group Agents. Canadian Journal of Philosophy. 2020;50(3):350364.

[26] Ritchie K. Social Structures and the Ontology of Social Groups. Philosophy and Phenomenological Research. 2020;100(2):402-424.

[27] Fine K. The Identity of Social Groups. Metaphysics. To appear.

[28] Ruben DH. Social Wholes and Parts. Mind. 1983;XCII(366):219-238.

[29] Ruben DH. The Metaphysics of the Social World. Routledge \& Kegan Paul; 1985.

[30] Mizoguchi R, Kitamura Y, Borgo S. A unifying definition for artifact and biological functions. Applied Ontology. 2016;11(2):129-154.

[31] Varzi A. Mereology. In: Zalta E, editor. The Stanford Encyclopedia of Philosophy. Spring 2019 ed. Stanford University; 2019.

[32] Masolo C, Borgo S, Gangemi A, Guarino N, Oltramari A. The WonderWeb Library of Foundational Ontologies and the DOLCE ontology - WonderWeb Deliverable D18. LOA-ISTC-CNR; 2003.

[33] Rector A, Rogers J, Bittner T. Granularity, scale and collectivity: when size does and does not matter. Journal of Biomedical Informatics. 2006;39(3):333-349.

[34] Guizzardi G. Ontological foundations for conceptual part-whole relations: The case of collectives and their parts. In: Mouratidis H, Rolland C, editors. Proceedings of the 23rd International Conference on Advanced Information Systems Engineering (CAiSE 2011). Springer; 2011. p. 138-153.

[35] Fine K. Things and Their Parts. Midwest Studies in Philosophy. 1999;XXIII(61-74).

[36] Masolo C, Benevides AB, Porello D. The interplay between models and observations. Applied Ontology. 2018;13(1):41-71.

[37] Fine K. Acts and Embodiment. In: Hinshelwood A, editor. Being and Doing. Oxford University Press; To appear.

[38] Oliver A, Smiley T. Multigrade predicates. Mind. 2004;113(452):609-681.

[39] Ferrario R, Masolo C, Porello D. Organisations and Variable Embodiments. In: Borgo S, Hitzler P, Kutz O, editors. Formal Ontology in Information Systems: Proceedings of the 10th International Conference (FOIS 2018). IOS Press; 2018. p. 127-140.

[40] Uzquiano G. Groups: Toward a Theory of Plural Embodiment. Journal of Philosophy. 2018;115(8):423452.

[41] Wilson R. A puzzle about material constitution and how to solve it: Enriching constitution views in metaphysics. Philosophers' Imprint. 2007;7(5).

[42] Galton A. How is a collection related to its members. In: Proceedings of the Third Interdisciplinary Ontology Meetings. Keio University; 2010. p. 9-17.

[43] Fine K. Towards a theory of part. The Journal of Philosophy. 2010;107(11):559-589.

[44] Porello D, Bottazzi E, Ferrario R. The Ontology of Group Agency. In: Garbacz P, Kutz O, editors. Proceedings of 8th International Conference on Formal Ontology and Information Systems (FOIS 2014). IOS Press; 2014. p. 183-196. 